\title{
NEW CORRELATIONS FOR PREDICTING THE TRANSIENT BEHAVIOR OF GRANULAR FILTRATION
}

\author{
JUN-ICHIRO TSUBAKI AND HIDETOSHI MORI \\ Department of Molecular Design and Engineering, University of \\ Nagoya, Nagoya 464
}

Key Words: Aerosol Filtration, Granular Bed Filter, Collection Efficiency, Particle Loading

\begin{abstract}
A single-parameter model has been developed for the prediction of transient behavior of granular filtration. The model is based on the experimental finding that the collection efficiency has a characteristic dependency on an increase in the pressure drop caused by retained deposits. Practical correlations are established relating the histories of filter performance with the extent of deposit and other relevant variables. It is demonstrated that the predicted results agree well with the experimental data conducted to determine the changes in effluent quality and the pressure drop necessary for the specified flow rate.
\end{abstract}

\section{Introduction}

Granular filtration in a fixed bed is inherently a time dependent process (Fichman et al., 1988, Tien, 1989, and Jung and Tien, 1991 and 1992). As the filtration process proceeds, the filter medium undergoes a continuous change in its structure with the accumulation of deposited particles. This change results in the transient behavior of the filter's particle collecting ability as well as the pressure drop necessary to maintain a given gas flow rate through the filter. Since a filter's performance is measured by its filtrate quality and the pressure drop often limits the duration of the filtration run, the ability to predict the histories of effluent concentration and pressure is a major concern in a practical consideration.

The parameter characterizing the particle collecting ability of a filter is the so-called filter coefficient, $\lambda$, which can be correlated with the unit collector efficiency. To account for the effect of particle deposition, Walata et al, (1986) proposed the expression

$$
\frac{\lambda}{\lambda_{0}}=F_{1}(\alpha, \sigma)=1+\alpha_{1} \sigma^{\alpha_{2}}
$$

where $\sigma$ is the specific deposit, which is a measure of the extent of particle deposition and defined as the volume of the particle deposited per unit filter volume. $\alpha_{1}$ and $\alpha_{2}$ are empirical constants and vary with operating variables. In the same manner as $F_{1}$, they expressed the change in filter resistance in terms of the ratio of the required pressure drop to that of a clean filter as

Received on July 25, 1996. Correspondence concerning this article should be addressed to J. Tsubaki.

$$
\frac{\Delta p}{\Delta p_{0}}=F_{2}(\beta, \sigma)=1+\beta_{1} \sigma^{\beta_{2}}
$$

where $\beta_{1}$ and $\beta_{2}$ are empirical constants. A major disadvantage of this model is that it contains several parameters which render it difficult to use without adequate experimental support. It should be noted that Eqs. (1) and (2) are essentially applicable for the bed with unit height, $l$, which is of the magnitude of grain size, as described later. In most experiments of granular filtration, however, we measure the transient behavior of filter performance using experimental filters of various types, the height of which may be several tenths of the unit collector height. In these cases, the parameters $\alpha$ and $\beta$ may be correlated with an average value of the deposit, $\sigma$, since the extent of deposition has nonuniformity throughout any filter.

In order to apply the empirical correlations of Eqs. (1) and (2) for the extraction of quantitative information on the effect of deposition on filter performance, Walata et al (1986) developed a method to determine the empirical parameters by a data reduction procedure. This method was subsequently applied by Takahashi et al. (1986) and by Michaels and Goren (1987) to relate these empirical constants with Stokes and interception parameters. Their works include a reasonably accurate estimation of the filter performance. However those who use this approach require the use of data sets obtained from filters of different height under similar conditions. It should be also noted that the time dependent behavior of filter performance is to be numerically predicted even though the empirical parameters are obtained from the filtration data. This, plus the fact that transient state experiments are usually lengthy, have made this approach laborious and difficult.

In the present study, an attempt has been made 
to establish a new correlation conveniently employed for predicting the effect of particle deposition on granular filtration. A single parameter model is deduced from the observation of filter performance histories and examined using available experimental data.

\section{Transient Behavior of Granular Filtration}

The phenomenological expressions which can describe the dynamic of granular filtration have been developed by Tien and Payatakes (1979) and Tien (1982). These expressions are based on the experimental finding that the particle concentration profile throughout a filter can often be described by the logarithmic law. The specific deposit is a local function and varies with time.

It is conceivable that the use of uniform deposition becomes acceptable when the bed is sufficiently thin. In the most general case, one may assume a filter bed to be an assembly of unit collectors connected in series, as shown in Fig. 1. The total number $n$ of unit bed elements in series for a filter of height $L$ is

$$
n=\frac{L}{l}
$$

where $l$ is the axial height for a unit bed element. For the constricted tube model, one has (Payatakes et al., 1973)

$$
l=\left\{\frac{\pi}{6(1-\varepsilon)}\right\}^{1 / 3} d_{\mathrm{g}}
$$

If $\eta_{i}$ denotes the collection efficiency of the $i$-th unit bed element, by definition, one has the overall collection efficiency $E_{i}$ and pressure drop $\Delta P_{i}$ (from the filter entrance through the $i$-th element) as

$$
\begin{aligned}
& 1-E_{i}=\prod_{j=1}^{i}\left(1-\eta_{j}\right) \\
& \Delta P_{i}=\sum_{j=1}^{i} \Delta p_{j}
\end{aligned}
$$

During the initial period of an experiment, the filter would be relatively free of deposited particles, and therefore all $\eta_{i}$ 's are the same. By rewriting Eq. (5) one can determine the initial collection efficiency from

$$
1-E_{i 0}=\left(1-\eta_{0}\right)^{i}
$$

where $\eta_{0}$ is the initial value of $\eta$.

If $C_{j}$ denotes the effluent particle concentration of the $j$-th unit bed element, the total number of

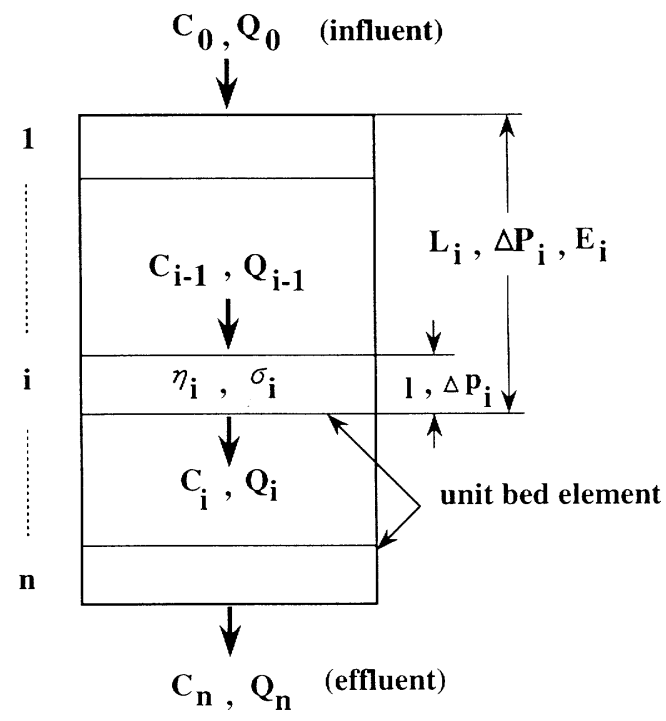

Fig. 1 Schematic representation of granular bed filter

particles entering the $j$-th element is

$$
Q_{j-1}=\int_{0}^{t} C_{j-1} u d t
$$

and the specific deposit is expressed as

$$
l \sigma_{j}=\left(Q_{j-1}-Q_{j}\right)
$$

From the overall mass balance of particles for the unit elements $(j=1 \sim i)$, one has

$$
\bar{\sigma}_{i}=\frac{1}{i} \sum_{j=1}^{i} \sigma_{j}
$$

and

$$
C_{0} u E_{i} d t=L_{i} d \bar{\sigma}_{i}
$$

\section{Experimental Work}

The apparatus used for the experimental work is based on the original design developed by Walata et al. (1986). The system is described in detail elsewhere (Walata et al, 1986 and Takahashi et al., 1986). Monodispersed polyvinyltoluene latex particles (diameter $d_{\mathrm{p}}=2.02 \mu \mathrm{m}$, Dow Chemical Co., Indianapolis, IN), the electrostatic charges of which were reduced with a neutralizer (Thermo Systems, Inc., Model 3021, St. Paul, MN), were used to prepare the test suspensions. Particle concentrations of both the influent and effluent samples were determined using a Climet Particle Counter (Clime Instrument Co., Model 208, Redland, CA).

Glass spheres (average diameter $d_{\mathrm{g}}=525 \mu \mathrm{m}$ ) within a narrow size range were used as filter grains. Measurements were carried out at constant filtration 
velocity of $11.3 \mathrm{~cm} / \mathrm{s}$ for the experimental filter, the packing height of which was adjusted to be 1.0, 2.0, 4.0 , and $8.0 \mathrm{~cm}$. The filter porosity was determined for each experiment and controlled so that the initial pressure drop increases proportionately with an increase in the bed height. The pressure drop across the filter was measured using a manometer.

\section{Results and Discussion}

A typical set of experimental results is shown in Fig. 2, in which the effluent-to-influent concentration, penetration $(1-E)$, is given as a function of the corresponding pressure drop. It is clearly demonstrated that the overall penetration yields linear plot versus pressure drop on a log-log graph, irrespective of the total bed height.

To gain insight into the performance of a filter, it is essential that the unit collector efficiency and the pressure drop across the unit bed element should be determined as a function of the specific deposit in a similar way as Eqs. (1) and (2) have been proposed. We assume the following equations to reduce the number of parameter used in Eqs. (1) and (2)

$$
\begin{aligned}
& \frac{1-\eta}{1-\eta_{0}}=e^{l \alpha \sigma} \\
& \frac{\Delta p}{\Delta p_{0}}=e^{\beta \sigma}
\end{aligned}
$$

where $\alpha$ and $\beta$ are empirical constants. Equation (12) is assumed on the basis of the logarithmic law describing the particle concentration profile throughout a filter, the law for which has been demonstrated in a previous paper (Mori et al., 1992) to be applicable in the prediction of the performance of granular bed filters with particle loading. While in the assumption of increase in pressure drop, we assume Eq. (13) which is approximately expressed as a series of $\sigma$ if the value of $\sigma$ is much smaller than unity. The resulting expanded expression would be considered to be a general format of Eq. (2). Equations (12) and (13) are used to deduce a relation of the collection efficiency of a filter with a given height to the pressure drop across the filter, the relation for which will lend reasonable support to the result demonstrated in Fig. 2. The present expression contains one adjustable parameter which may vary with operating variables. To demonstrate the validation of the above format, correlations in predicting the transient behavior of granular filtration are developed in the sections below.

\subsection{Correlations for filter collection efficiency}

The increase in collection efficiency may be characterized by the extent of deposition. Average specific deposit can be correlated with $E_{i}$ by substituting Eqs. (9) and (10) into Eq. (12), and yields

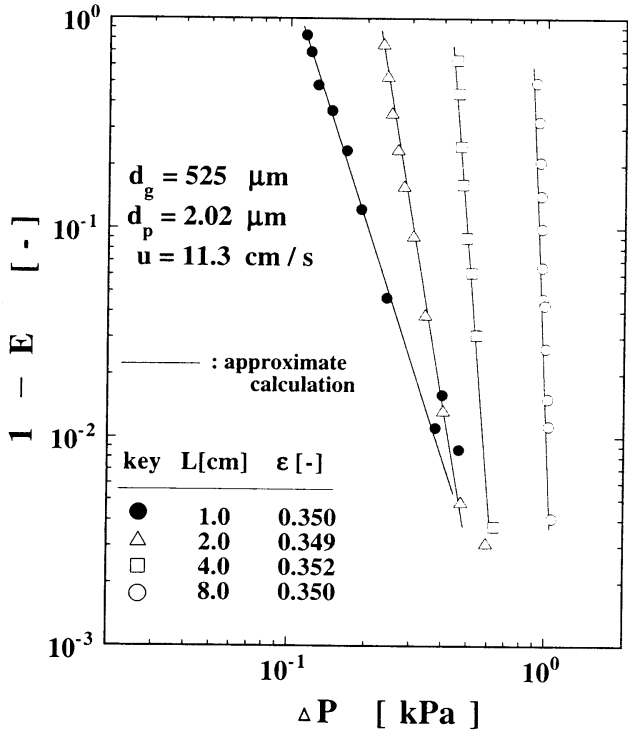

Fig. 2 Transient behavior of collection efficiency and pressure drop

the resulting format with help of Eqs. (5) and (7)

$$
\frac{1-E_{i}}{1-E_{i 0}}=e^{\alpha L_{i} \bar{\sigma}_{i}}
$$

Equation (14) shows that the determinations of $\eta_{j}$ and $\sigma_{j}$ are not necessarily required in order to estimate the value of $\alpha$, which is really determined from the data of overall transient behavior of granular filtration. This fact makes the present model more convenient than the two-parameter model expressed by Eqs. (1) or (2).

Substituting the time-derivative of Eq. (14) into Eq. (11), one has time changes in overall collection efficiency, which is finally related to the total number of particles entering the filter during the filtration run, or

$$
\frac{E_{i 0}\left(1-E_{i}\right)}{E_{i}\left(1-E_{i 0}\right)}=e^{\alpha Q_{0}}
$$

The filtration behavior corresponding to one set of conditions, along with the results calculated by Eq. (14), is shown in Fig. 3 in the form of 1-E vs. $\bar{\sigma}$. The empirical constant $\alpha$ used for the prediction was obtained by the experiment for the filter with $L=1.0 \mathrm{~cm}$, in which case the use of uniform deposition assumption proved to be reasonably acceptable (Jung and Tien, 1991). Equation (15) is compared with the observed data in Fig. 4, where $(1-E) / E$ is plotted against the total number of particles entering the filter. Figure 5 shows a comparison of Eq. (14) with the data available in the literature (Takahashi et al., 1986), in which they measured the time dependence of the 


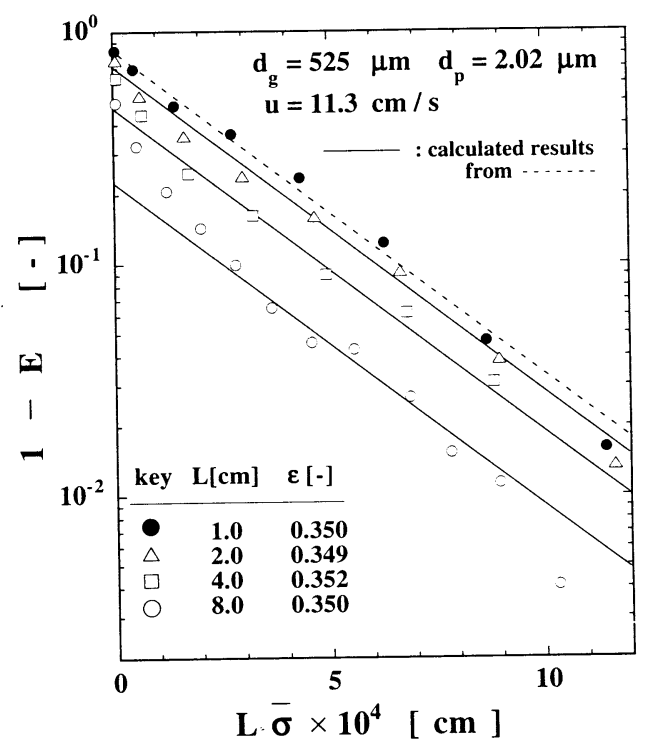

Fig. 3 Overall penetration versus average specific deposit

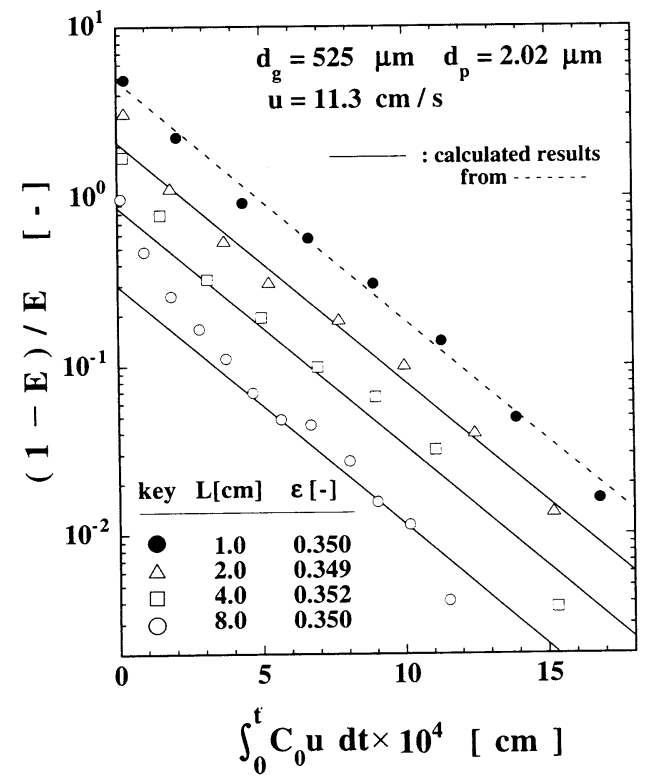

Fig. 4 Relation of $(1-E) / E$ to total number of particles entering filter

penetration of $2.02 \mu \mathrm{m}$-diameter latex aerosols through granular beds composed of 99 or $266 \mu \mathrm{m}$ diameter glass spheres. The experimental filter was thin enough to assume uniform deposition. As shown in the figure, the total penetration (1-E) decreases straightly with increasing specific deposit on a semi-log graph, the result of which reasonably supports Eq. (14).

Figures 3 to 5 demonstrate that the present model successfully predicts the transient behavior of the collection efficiency within a certain limit of filter

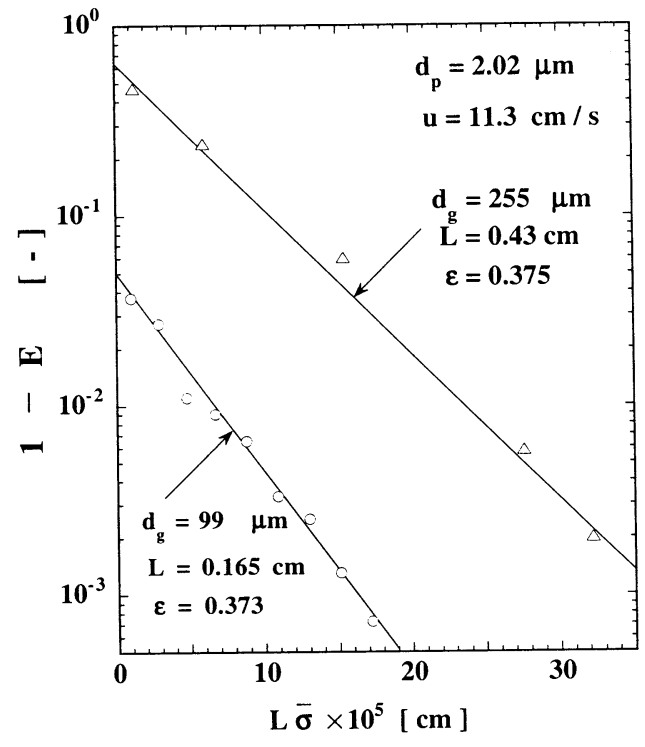

Fig. 5 Overall penetration versus average specific deposit (data reduced from Ref. (Takahashi et al., 1986))

height. When the filter height becomes greater, a difference exists particularly in the initial period of the filtration process, where the correlation tends to overestimate. Although this result suggests that the value of the empirical constant $\alpha$ may depend on the filter height, the prediction would be accurate enough for practical usage (Mori et al., 1992).

The average collection efficiency defined as

$$
\bar{E}_{i}=\frac{L_{i} \bar{\sigma}_{i}}{Q_{0}}
$$

can be readily given from Eqs. (14) and (15) by eliminating $E_{i}$

$$
\bar{E}_{i}=1-\frac{1}{\alpha Q_{0}} \ln \left\{E_{i 0}+\left(1-E_{i 0}\right) e^{\alpha Q_{0}}\right\}
$$

This format is equal to the following expression proposed by Mori et al. (1992)

$$
\bar{E}_{i}=\ln \frac{1-E_{i}}{1-E_{i 0}} \mid \ln \frac{E_{i 0}\left(1-E_{i}\right)}{E_{i}\left(1-E_{i 0}\right)}
$$

The above expression is also deduced from Eq. (17) by combining Eqs. (15) and (17) to express the average collection efficiency as a function of $E_{j 0}$ and $E_{j}$. They demonstrated the validity of Eq. (18) by experimental work using various types of filters, the results of which support the single-parameter model proposed in the present paper.

\subsection{Correlations for pressure drop}

The total pressure drop is equal to the sum of the pressure drop across the unit collectors comprising the 
bed. When the assumption that $\beta \sigma \ll 1$ is reasonably acceptable, one has the pressure drop across the $j$-th unit bed element from Eq. (13) as

$$
\Delta p_{j}=\Delta p_{0} e^{\beta \sigma_{j}} \cong \Delta p_{0}\left(1+\beta \sigma_{j}\right)
$$

The total pressure drop (Eq. (6)) is expressed accordingly as

$$
\frac{\Delta P_{i}}{\Delta P_{0}}=e^{\beta \bar{\sigma}_{i}}
$$

Equations (20) and (14) combine to give the collection efficiency with the corresponding pressure drop.

$$
\frac{1-E_{i}}{1-E_{i 0}}=\left(\frac{\Delta P_{i}}{\Delta P_{i 0}}\right)^{L_{i} \frac{\alpha}{\beta}}
$$

The above relation reasonably supports the result shown in Fig. 2, where the overall penetration of a given filter yields linear plot versus pressure drop on a $\log -\log$ graph. By eliminating 1- $E_{i}$ from Eqs. (15) and (21), the transient behavior of pressure drop is found to be

$$
\frac{\Delta P_{i}}{\Delta P_{i 0}}=\left\{\frac{e^{\alpha Q_{0}}}{\left(1-E_{i 0}\right)\left(e^{\alpha Q_{0}}-1\right)+1}\right\}^{\frac{\beta}{L_{i \alpha}}}
$$

The predicted behaviors of pressure drop are shown in Figs. 6 and 7 in the form of $\Delta P$ vs. $\bar{\sigma}$ and $\Delta P$ vs. $Q_{0}$, respectively. The empirical constants $\alpha$ and $\beta$ were determined from the results for the experimental filter with $L=1.0 \mathrm{~cm}$. The dot-dash-lines in the figures represent the results approximately calculated using the assumption of Eq. (19). Whilst the solid lines show the results exactly calculated by applying Eq. (13) to each unit bed element. As the filtration process proceeds, the extent of deposition becomes significant, suggesting that the assumption of Eq. (19) does not hold any longer. Figures 6 and 7 demonstrate that the present model (Eq. (13)) successfully predicts the transient behavior for the increase in pressure drop for different heights of filters.

According to the definition of $\sigma_{i}$, one has

$$
\sigma_{i}=i \bar{\sigma}_{i}-(i-1) \bar{\sigma}_{i-1}
$$

Equations (16) and (17) combine to give the average specific deposit as

$$
\bar{\sigma}_{i}=\frac{1}{L_{i} \alpha} \ln \frac{e^{\alpha Q_{0}}}{\left(1-E_{i 0}\right)\left(e^{\alpha Q_{0}}-1\right)+1}
$$

The profile of specific deposit within a bed is therefore correlated with $Q_{0}$ by substituting Eq. (24) into Eq.

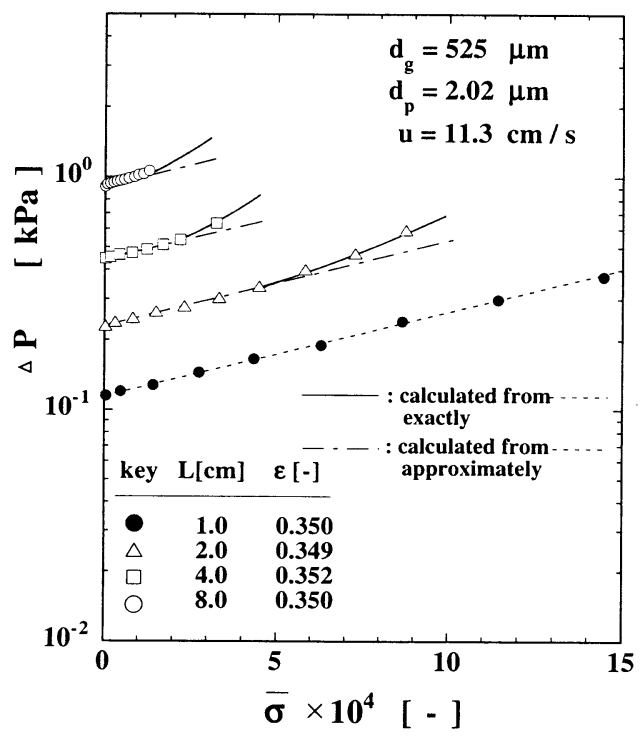

Fig. 6 Pressure drop data across filter versus average specific deposit

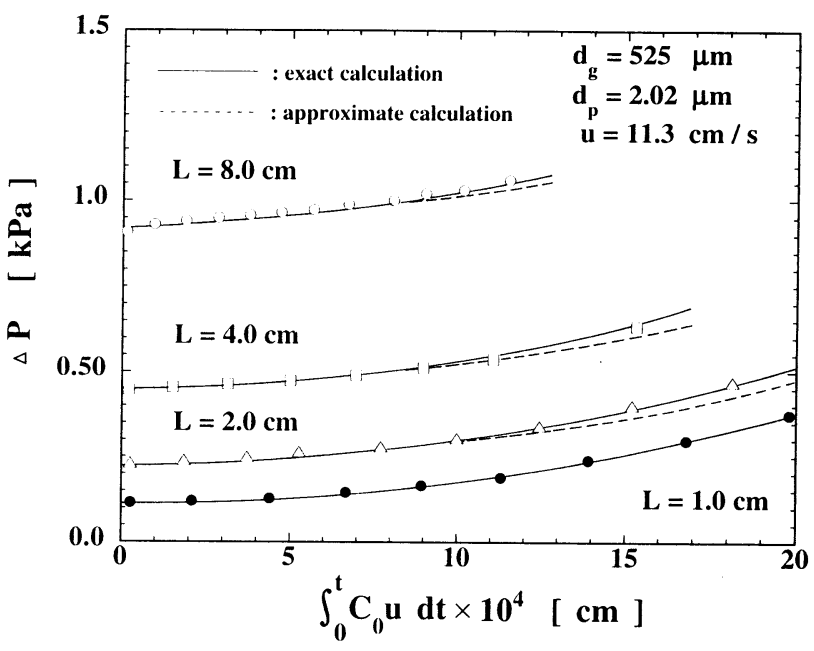

Fig. 7 Pressure drop data and their comparisons with predictions

(23), and the resulting expression is

$$
\sigma_{i}=\frac{1}{\alpha l} \ln \frac{\left(1-\eta_{0}\right)^{i-1}\left(e^{\alpha Q_{0}}-1\right)+1}{\left(1-\eta_{0}\right)^{i}\left(e^{\alpha Q_{0}}-1\right)+1}
$$

The calculated results are shown in Fig. 8, where the specific deposit at each unit bed element is plotted at seven $Q_{0}$ 's as indicated. As the filtration proceeds, the deposit near the filter entrance tends to rapidly increase. If the granular filtration is prolonged furthermore, the filter becomes increasingly clogged and then surface filtration would start as the dominant filtration mechanism for the entering particles. When surface filtration becomes dominant, the collection efficiency of a filter will approach unity but 


\section{L $[\mathbf{c m}]$}

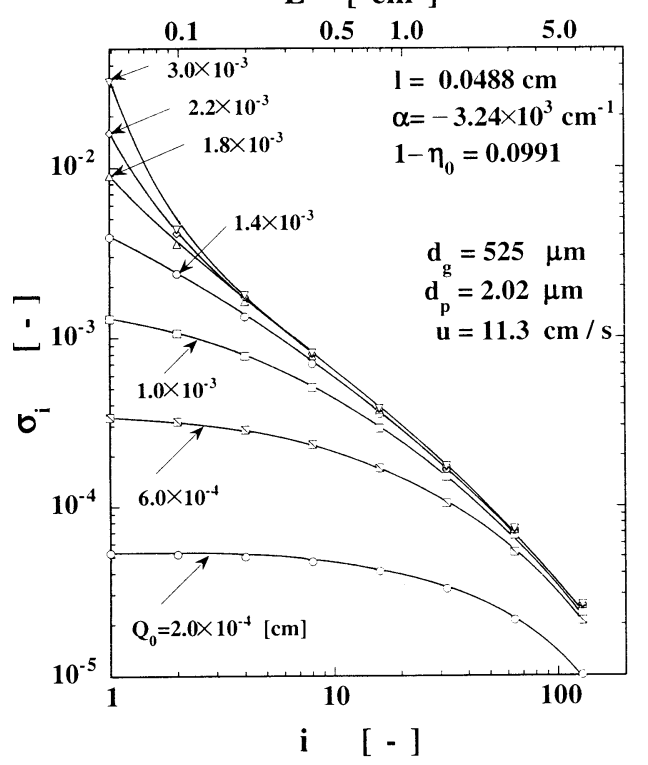

Fig. 8 Predicted time changes in deposit profile through filter

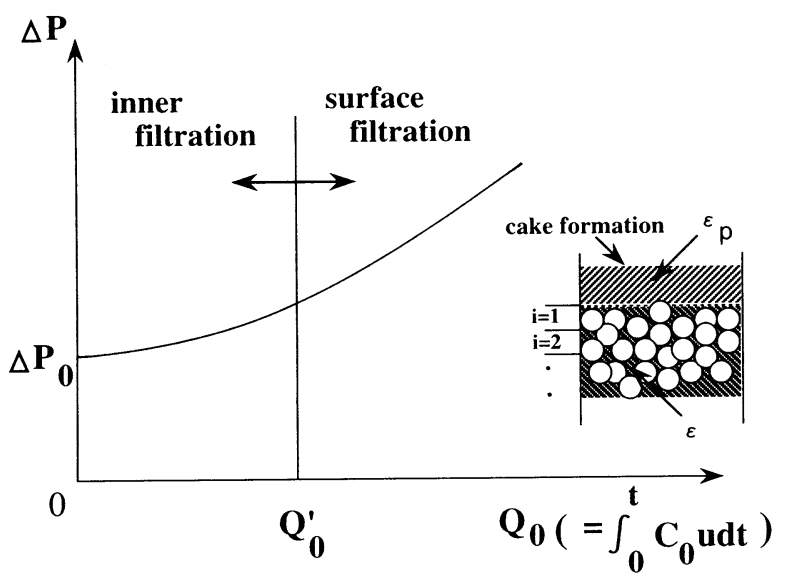

Fig. 9 Schematic pressure drop versus inlet particle load illustrating different filtration regimes

the pressure drop would keep on increasing continuously, thus forming a filter cake on the bed, as illustrated in Fig. 9. If we assume that the porosity of the formed cake should be equal to that of the particle deposit within the filter, the inner filtration process would continue until the specific deposit at the first unit bed element $(i=1)$ satisfies the following condition

$$
\sigma_{1}=\left(1-\varepsilon_{\mathrm{p}}\right) \varepsilon
$$

The filtration time, more precisely the total number of particles entering the filter $Q_{0}{ }^{\prime}$, required to satisfy Eq. (26) is also predicted using Eq. (25). After the filtration time of $Q_{0}^{\prime}$, the filter cake with a porosity of

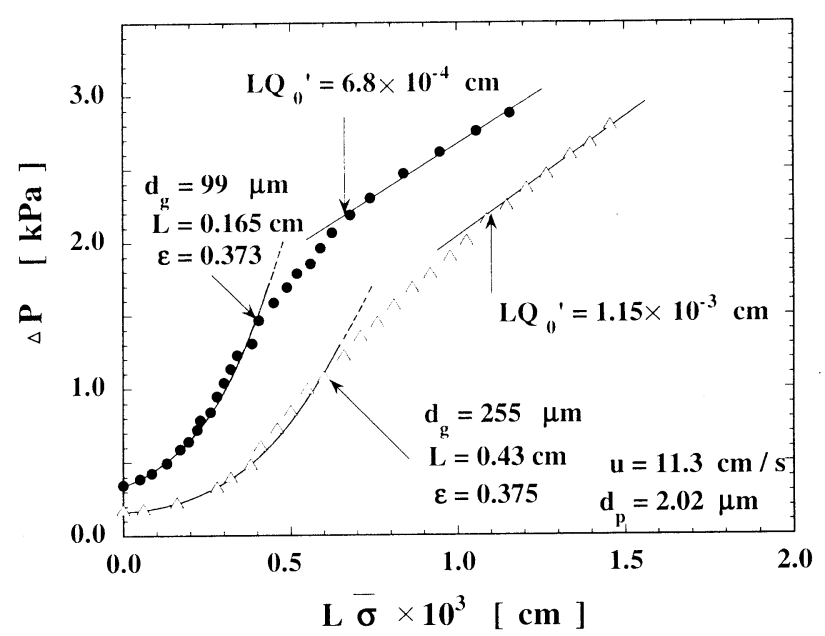

Fig. 10 Prediction of deposit porosity from data on the history of pressure drop (data reduced from Ref. (Takahashi et al., 1986))

$\varepsilon_{\mathrm{p}}$ grows continuously and the pressure drop, being in proportion to the amount of particles entering the filter, would increases accordingly.

The deposit porosity $\varepsilon_{\mathrm{p}}$ was estimated from the data measured by Takahashi et al. (1986). They measured time dependence of the pressure drop across the filter over a long period of operation. The results are shown in Fig. 10 in the form of $\Delta P$ vs. $L \bar{\sigma} . \quad$ In the region of inner filtration mechanism, the pressure drop can be precisely predicted by applying Eq. (13) to each unit bed element. After the transition region, the pressure drop shows a tendency to increase linearly with increasing deposit, suggesting that surface filtration should become the dominant mechanism. As shown in Fig. 10, the value of $L \bar{\sigma}$ from which the pressure drop would start to increase straightly can be obtained by drawing a straight line passing through the points of data measured under the high load conditions. The filtration time, and hence the total number of particles entering the filter $Q_{0}{ }^{\prime}$, required for the deposition of $\bar{\sigma}$ is obtained using Eq. (24). The deposit porosity which satisfies Eq. (26) is then determined from Eq. (25). From the data in Fig. 10 we estimate the value of $\varepsilon_{\mathrm{p}}$ to be 0.83 for the filter composed of $99 \mu \mathrm{m}$-diameter grains, and 0.94 for the filter of $255 \mu \mathrm{m}$-diameter grains. The deposit porosity, of course, depends on the morphology of the deposits formed, and hence varies with the operation variables.

\section{Conclusion}

Experiments have been conducted to determine the twin features of transient behavior of fixed-bed granular filtration, the results of which reasonably lend support to derive the single-parameter model. 
The present model contains one adjustable parameter which can be readily determined from a single experiment under a given set of conditions.

Available correlations are established relating filter performance with the extent of deposits and other relevant variables. It has been demonstrated that the availability of those correlations makes it possible to predict the performance of fixed granular bed filters. Time changes in collection efficiency and also in the profile of deposits within a filter are predicted with enough accuracy by assuming a filter bed to be an assembly of unit collectors connected in series. Predictions of time changes in pressure drop through a filter agree well with the experimental data measured in the region where the inner filtration is dominant as a filtration mechanism. It has been shown that the deposit porosity can be determined from the filtration time from which the pressure drop would start to increase straightly, based on the assumption that the porosity of the formed cake should be equal to that of the deposits.

\section{Acknowledgment}

The work reported here was conducted in part at the Department of Chemical Engineering and Materials Science, Syracuse University, NY. Here the authors would like to express their appreciation to Prof. C. Tien.

\begin{tabular}{|c|c|c|c|}
\hline \multicolumn{4}{|c|}{ Nomenclature } \\
\hline C & $=$ & particle volume concentration in fluid & [-] \\
\hline$C_{0}$ & $=$ & influent particle concentration & [-] \\
\hline$C_{i}$ & $=$ & $\begin{array}{l}\text { concentration of effluent of } i \text {-th unit bed } \\
\text { element }\end{array}$ & \\
\hline$d_{\mathrm{g}}$ & $=$ & grain diameter & {$[n$} \\
\hline$d_{\mathrm{p}}$ & $=$ & diameter of aerosol particle & \\
\hline E & $=$ & overall collection efficiency & {[} \\
\hline$E_{i}$ & $=$ & $\begin{array}{l}\text { collection efficiency of bed consisting of } 1 \sim i \\
\text { unit bed elements }\end{array}$ & $-\mathrm{th}$ \\
\hline$E_{i 0}$ & $=$ & initial value of $E_{i}$ & {$[-$} \\
\hline $\bar{E}_{i}$ & $=$ & average collection efficiency defined by Eq. ( & \\
\hline & & & {$[-$} \\
\hline$L$ & $=$ & filter height & {$[\mathrm{m}$} \\
\hline$L_{i}$ & $=$ & $\begin{array}{l}\text { height of bed consisting of } 1 \sim i \text {-th unit bed } \\
\text { elements }\end{array}$ & {$[\mathrm{m}$} \\
\hline$l$ & $=$ & axial distance of unit bed element & {$[\mathrm{m}$} \\
\hline$n$ & $=$ & number of unit bed elements in a filter & {$[-$} \\
\hline$Q$ & $=$ & $\begin{array}{l}\text { total number of particles entering a filter or u } \\
\text { bed element }\end{array}$ & \\
\hline$Q_{0}$ & $=$ & total number of particles entering a filter & {$[\mathrm{m}$} \\
\hline$Q_{i}$ & & $\begin{array}{l}\text { total number of particles exiting } i \text {-th unit } \\
\text { bed element }\end{array}$ & \\
\hline$u$ & $=$ & filtration velocity & {$[\mathrm{m} / \mathrm{s}$} \\
\hline$\alpha, \beta$ & $=$ & $\begin{array}{l}\text { empirical constants defined by Eqs. (12) an } \\
\text { respectively }\end{array}$ & \\
\hline
\end{tabular}

\begin{tabular}{|c|c|c|c|}
\hline$\alpha_{1}, \alpha_{2}$ & & empirical constants used in Eq. (1) & [-] \\
\hline$\beta_{1}, \beta_{2}$ & $=$ & empirical constants used in Eq. (2) & [-] \\
\hline$\Delta P$ & $=$ & pressure drop & {$[\mathrm{Pa}]$} \\
\hline$\Delta P_{0}$ & $=$ & initial value of $\Delta P_{i}$ & {$[\mathrm{~Pa}]$} \\
\hline$\Delta P_{i}$ & $=$ & $\begin{array}{l}\text { pressure drop across bed consisting of } 1 \sim i \text {-th } \\
\text { unit bed elements }\end{array}$ & {$[\mathrm{Pa}$} \\
\hline$\Delta p$ & $=$ & pressure drop across a unit bed element & \\
\hline$\Delta p_{0}$ & $=$ & initial value of $\Delta p_{i}$ & \\
\hline$\Delta p_{i}$ & $=$ & pressure drop across $i$-th unit bed element & \\
\hline$\varepsilon$ & $=$ & filter bed porosity & \\
\hline$\varepsilon_{\mathrm{p}}$ & $=$ & porosity of deposits & \\
\hline$\eta$ & $=$ & unit collector efficiency & \\
\hline$\eta_{0}$ & $=$ & initial value of $\eta_{i}$ & \\
\hline$\eta_{i}$ & $=$ & $\begin{array}{l}\text { unit collector efficiency of } i \text {-th unit bed } \\
\text { element }\end{array}$ & \\
\hline$\lambda$ & $=$ & filter coefficient & \\
\hline$\sigma$ & $=$ & specific deposit & \\
\hline$\sigma_{i}$ & $=$ & specific deposit of $i$-th unit bed element & \\
\hline $\bar{\sigma}$ & $=$ & average specific deposit & \\
\hline $\bar{\sigma}_{i}$ & $=$ & average value of $\sigma_{i}$, defined by Eq. (10) & \\
\hline
\end{tabular}

\section{Literature Cited}

Fichman, M., C. Gutfinger and D. Pnueli; "Effect of Particle Loading on Granular Bed Filtration-The Cluster Enhanced Model," J. Aerosol Sci., 19, 425-441 (1988a)

Fichman, M., C. Gutfinger and D. Pnueli; "Effect of Particle Loading on Granular Bed Filtration-Extension of the CEF Model to Polydisperse Systems," J. Aerosol Sci., 19, 443-450 (1988b)

Jung, Y. and C. Tien; "New Correlations for Predicting the Effect of Deposit on Collection Efficiency and Pressure Drop in Granular Filtration," J. Aerosol Sci., 22, 187-200 (1991)

Jung, Y. and C. Tien; "Increase in Collector Efficiency Due to Deposition in Polydispersed Granular Filtration -an Experimental Study," J. Aerosol Sci., 23, 525-537 (1992)

Michaels, C, and S.L. Goren; "Aerosol Capture in Particle Laden Granular Beds in the Impaction Dominated Regime," Aerosol Sci. Technol., 7, 31-46 (1987)

Mori, H., N. Kimura and S. Toyama; "The Performance of Granular Bed Filters with Dust Loading," J. Soc. Powder Technol., Japan, 29, 18-25 (1992)

Payatakes, A.C., R.M. Turian and C. Tien; "A New Model for Granular Porous Media-Model Formation,” AIChE J., 19, 5867 (1973)

Takahashi, T., S.A. Walata and C. Tien; "Transient Behavior of Granular Filtration of Aerosols-Effect of Aerosol Deposition on Filter Performance," AIChE J., 32, 684-690 (1986)

Tien, C.; Granular Filtration of Aerosols and Hydrosols, p. $303-$ 340, Butterworth, Boston, U.S.A. (1989)

Tien, C.; "Aerosol Filtration in Granular Media," Chem. Eng. Commun., 17, 361-382 (1982)

Tien, C. and A.C. Payatakes; "Advances in Deep Bed Filtration," AIChE J., 25, 737-759 (1979)

Walata, S.A., T. Takahashi and C. Tien; "Effect of Particle Deposition on Granular Aerosol Filtration-A Comparative Study of Methods in Evaluating and Interpreting Experimental Data," Aerosol Sci. Technol., 5, 23-37 (1986) 